\title{
Disease Response Sponsor Defined Identifier
}

National Cancer Institute

\section{Source}

National Cancer Institute. Disease Response Sponsor Defined Identifier. NCI Thesaurus.

Code C117401.

One or more sponsor defined characters used to identify, name, or characterize the disease response assessment. 